\title{
Two-dimensional problem of thermoelectric materials with an elliptic hole or a
}

\section{rigid inclusion}

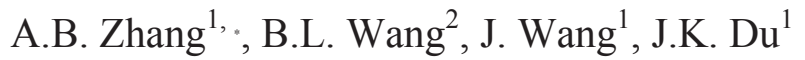 \\ ${ }^{1}$ Piezoelectric Device Laboratory, School of Mechanical Engineering \& Mechanics, \\ Ningbo University, Ningbo, 315211, P.R. China \\ ${ }^{2}$ Centre for Infrastructure Engineering, Western Sydney University, Penrith, NSW \\ 2751, Australia
}

\begin{abstract}
The two-dimensional problems of an elliptic hole or a rigid inclusion embedded in a thermoelectric material subjected to uniform electric current density and energy flux at infinity are studied based on the complex variable method of Muskhelishvili and conformal mapping technique. The closed-form solutions of electric potential, temperature and stress components are presented according to electrical insulated and thermal exact boundary conditions on the rim of the hole or inclusion. Numerical results are carried out to illustrate the influence of the value of major to minor axis ratio of the elliptic geometry and heat conductivity of inhomogeneity on thermoelectric and stress fields. It is found that energy flux at surfaces of the hole or rigid inclusion does not vanish due to the Joule heat and Seebeck effect when the electric field is applied. In addition, stress induced by applied electric field has a non-linear relationship with the electric current density. The heat conductivity of the air inside the elliptic hole reduces the concentration factors of energy flux and stress. However, the concentration factors of energy flux and stress at the bonding interface increase with the increasing values of heat conductivity of the flat rigid inclusion.
\end{abstract}

Keywords: Thermoelectric material; Elliptic hole; Inclusion; Fracture mechanics

Corresponding author. Tel.: +8675487600980

E-mail address: zhangaibing@,nbu.edu.cn (A.B. Zhang). 


\section{Introduction}

Thermoelectric materials for solid state energy conversion have been extensively used for electric cooling [1], electricity power generation in harvesting wasted heat [2], solar energy harvesting [3] and carbon reduction [4]. However, most thermoelectric materials are brittle in nature with low fracture strength and toughness [5]. Thermoelectric materials are subjected to significant stress induced by thermal gradient, thermal shock and externally applied mechanical loadings under in-service particularly when used in waste heat recovery [6]. Defects resulting from the manufacturing processes, such as cracks and holes, can cause electric potential and temperature discontinuities across the defects boundary and stress concentrations, then result in fracture [7-13]. Fracture problems in thermoelectric materials have been received much attention and investigated by many experts recently, like other structural materials subjected heat loadings problems [14-16]. The slow Vickers indentation crack growth behavior of $\mathrm{Mg}_{2} \mathrm{Si}$ thermoelectric material was observed by Schmidt et al. [17]. Analytic solutions for the thermoelectric material containing a 2D crack problem were derived by Zhang and Wang [18], and Song et al. [19], respectively. It is found that electric current density, heat flux and stress exhibit traditional inverse square-root singularity at the crack front. Li et al. [20] examined the brittle failure behavior of $\mathrm{CoSb}_{3}$ based skutterudite thermoelectric material by using large-scale molecular dynamics simulations. Based on the complex variable method, the study dealing with thermal stress concentration induced by an elliptic hole has been performed by Zhang and Wang [21]. In above-mentioned works, the boundary conditions on the hole or crack surfaces are always supposed to be electrical and thermal impermeable. The thermal conductivity of crack interior has great influences on the mode-II stress intensity factor for a thermal-medium crack in a 
thermoelastic material under a thermal loading [22]. Zhang and Wang [23] discussed the applicability of crack surfaces thermal boundary condition in a layered thermoelectric or metal/thermoelectric material, and the results show that the heat conductivity of air filled in a crack cannot be neglected.

On the other hand, in order to meet the various technology applications, much effort on thermoelectric materials has been devoted towards improving their figure of merit and energy conversion efficiency [24-29]. The efficiency of thermoelectric conversion depends on a dimensionless figure of merit $Z T=\varepsilon^{2} \gamma T / k$ [30], where $\gamma$, $k, \varepsilon$ and $T$ are the electric conductivity, heat conductivity, Seebeck coefficient and absolute temperature, respectively. Thermoelectric material with high energy conversion efficiency requires not only high Seebeck coefficient to have high voltage output, but also high electric conductivity to reduce Joule heat loss and low heat conductivity to maintain large temperature difference. However, it is rather difficult to increase $Z T$ because of competing effects of electrical and thermal conductivities, that is, a good heat conductor is usually a good electric conductor as well. One of the main strategies in developing thermoelectric materials with high energy conversion efficiency is to engineer hybrid composites, especially with nanostructures. Comparing with the system with the single material, composite structures with the layered and/or inclusions have better thermal and mechanical behaviors [31-34]. The thermoelectric properties of semiconductor materials with nanoinclusions can be improved based on the concept of band bending at inclusion/semiconductor interfaces as an energy filter for electrons [35]. An effective path to increase the electrical conductivity while to decrease the thermal conductivity, and thus to improve the figure of merit by nano-inclusions was reported by Wang et al., [36]. Introduction of metallic inclusion can enhance the thermoelectric material performance of manganese 
silicide nanocomposites [37]. The presence of inclusions affects the energy conversion efficiency, strength and reliability of thermoelectric materials, and it is therefore important to study the local fields induced by the inclusions. Thermoelectric materials have increasing applications in the engineering, however, to our best knowledge relatively little work has been done for the inclusion problem based on the continuum mechanics theory.

In view of the above literature analysis, the purpose of this paper is to seek a general solution to the problem of a 2D thermoelectric material with an elliptic hole or rigid inclusion. The influence of heat conductivity of air inside the hole and inclusion on the distribution of electric field, temperature and stress is also investigated. The paper is organized as follows. Firstly, basic equations for thermoelectric material are outlined in Section 1. Next, closed-form solutions of the electric potential, temperature and stress fields for an elliptic hole and rigid inclusion are derived in Sections 3 and 4, respectively. Some numerical results are given in Section 5. Finally, Concluding remarks are made.

\section{Basic equations for thermoelectric materials}

We consider an isotropic thermoelectric material in which the electric potential is $V$ and the absolute temperature is $T$. Such a material is characterized by the electric conductivity $\gamma$, thermal conductivity $k$ and Seebeck coefficient $\varepsilon$. The governing equations for a thermoelectric material in the absence of electric charges and heat sources can be presented in the form $[38,39]$,

$$
\begin{gathered}
\nabla \cdot \mathbf{j}_{e}=0 \\
\nabla \cdot \mathbf{q}+\mathbf{j}_{e} \cdot \nabla V=0
\end{gathered}
$$

and the transport of electric current density vector $\mathbf{j}_{e}=\left[j_{e x}, j_{e y}\right]^{T}$ and heat flux 
vector $\mathbf{q}=\left[q_{x}, q_{y}\right]^{T}$ is given as

$$
\begin{gathered}
\mathbf{j}_{e}=-\gamma \nabla V-\gamma \varepsilon \nabla T \\
\mathbf{q}=-\gamma \varepsilon T \nabla V-\left(k+\gamma \varepsilon^{2} T\right) \nabla T
\end{gathered}
$$

Notice that the uncoupled transport equations of electricity and heat are recovered when $\varepsilon=0$, and the temperature function $T$ and its gradient $\nabla T$ enter into the heat transport Eq. (2)2, making the coupling nonlinear. Since energy is transported by both electrons and heat, the energy flux vector $\mathbf{j}_{u}=\left[j_{u x}, j_{u y}\right]^{T}$ can be derived from the electric current density and heat flux as $\mathbf{j}_{u}=\mathbf{q}+\mathbf{j}_{e} V$. For the sake of convenience, an analytic function $F$ is defined as $F=V+\varepsilon T[18]$, then we have

$$
\begin{gathered}
\mathbf{j}_{e}=-\gamma \nabla F \\
\mathbf{j}_{u}=-\gamma F \nabla F-k \nabla T
\end{gathered}
$$

Combining Eqs. (1) and (3), the constitutive equations become,

$$
\begin{gathered}
\nabla^{2} F=0 \\
k \nabla^{2} T+\gamma(\nabla F)^{2}=0
\end{gathered}
$$

For the two-dimensional thermoelectric problem considered here, the general solutions of thermo-electro-elastic fields are derived detailed in our previous work [21], and summarized briefly as follows. The solutions of $F$ and $T$ can be expressed as,

$$
\begin{gathered}
F=\operatorname{Re}\left[f_{1}(z)\right] \\
T=\operatorname{Re}[g(z)]-\frac{\gamma}{4 k} f_{1}(z) \overline{f_{1}(z)}
\end{gathered}
$$

where $z=x+i y, f_{1}(z)$ and $g(z)$ are unknown analytic functions and "Re" stands for the real part of a complex number. Re-writing Eq. (3) gives:

$$
\begin{gathered}
j_{e x}-i j_{e y}=-\gamma f_{1}^{\prime}(z) \\
j_{u x}-i j_{u y}=-\frac{\gamma}{2} f_{1}(z) f_{1}^{\prime}(z)-k g^{\prime}(z)
\end{gathered}
$$


The boundary conditions of electric current density and energy flux are,

$$
\begin{gathered}
f_{1}(z)-\overline{f_{1}(z)}=-\frac{2 i}{\gamma} \int j_{\text {en }}(s) d s+\text { constant } \\
\operatorname{Im}\left[\frac{\gamma}{4} f_{1}^{2}(z)+k g(z)\right]=-\int j_{u n}(s) d s+\text { constant }
\end{gathered}
$$

where "Im" stands for the imaginary part of a complex number, $j_{e n}(s)$ and $j_{u n}(s)$ represent the normal electric current density and normal energy flux for the point $s$ along the boundary, respectively.

The corresponding thermal stresses and displacement induced by the temperature field $T$ can be obtained as $[21,40]$ :

$$
\begin{gathered}
\sigma_{x x}+\sigma_{y y}=4 \operatorname{Re}\left[\varphi^{\prime}(z)\right]+\frac{2 \mu \alpha^{*} \gamma}{k(\kappa+1)} f_{1}(z) \overline{f_{1}(z)} \\
\sigma_{y y}-\sigma_{x x}+2 i \sigma_{x y}=2\left[\bar{z} \varphi^{\prime \prime}(z)+\phi^{\prime}(z)\right]+\frac{2 \mu \alpha^{*} \gamma}{k(\kappa+1)} f_{2}(z) \overline{f_{1}^{\prime}(z)} \\
2 \mu\left[u_{x}+i u_{y}\right]=\kappa \varphi(z)-z \overline{\varphi^{\prime}(z)}-\overline{\phi(z)}+2 \mu \alpha^{*} \int g(z) d z-\frac{\mu \alpha^{*} \gamma}{k(\kappa+1)} f_{2}(z) \overline{f_{1}(z)}
\end{gathered}
$$

where $f_{2}^{\prime}(z)=f_{1}(z), \varphi(z)$ and $\phi(z)$ are complex stress potential functions to be determined, $\mu$ is the shear modulus, and $\kappa$ and $\alpha^{*}$ are defined as follows,

$$
\begin{gathered}
\kappa=\left\{\begin{array}{cc}
\frac{3-v}{1+v}, & \text { for plane stress state } \\
3-4 v, & \text { for plane strain state }
\end{array}\right. \\
\alpha^{*}=\left\{\begin{array}{cc}
\alpha, & \text { for plane stress state } \\
(1+v) \alpha, & \text { for plane strain state }
\end{array}\right.
\end{gathered}
$$

$v$ and $\alpha$ are the Poisson's ratio and the linear thermal expansion, respectively. The resultant force between two points in an elastic body has the form of

$$
\varphi(z)+z \overline{\varphi^{\prime}(z)}+\overline{\phi(z)}=i \int\left(p_{x}+i p_{y}\right) d s-\frac{\mu \alpha^{*} \gamma}{k(\kappa+1)} f_{2}(z) \overline{f_{1}(z)}+\text { constant }
$$


where $p_{x}$ and $p_{y}$ are the external forces applied to the boundary. It should be noted that the main aim of this paper is to discuss mechanical behavior of thermoelectric materials subjected to thermoelectric loads, therefore, the constant terms $p_{x}$ and $p_{y}$ are taken as zero.

\section{The solution to an elliptic hole}

As shown in Fig.1, the infinite thermoelectric material with an elliptic hole in the $z$-plane can be mapped into the infinite region inside of a unit circle in the $\zeta$-plane $(\zeta=\xi+i \eta)$ by the complex mapping function $z=w(\zeta)$ :

$$
w(\zeta)=R\left(m \zeta+\frac{1}{\zeta}\right)
$$

with $R=\frac{a+b}{2}, m=\frac{a-b}{a+b}$, where $a$ and $b$ are length of the semiaxes of the ellipse on the $x$ and $y$ axes, respectively. The thermoelectric material is subjected to combined an uniform electric current density $j_{e 0}$ and an uniform energy flux $j_{u 0}$ at infinite, i.e.,

$$
\begin{aligned}
& j_{e x}=0, j_{e y}=j_{e 0} \\
& j_{u x}=0, j_{u y}=j_{u 0}
\end{aligned} \quad \text { as } \quad \sqrt{x^{2}+y^{2}} \rightarrow \infty
$$

In addition, the hole is assumed to be free of tractions and filled with air. The electric conductivity of air inside the hole can be neglected since it is almost $10^{16} \sim 10^{18}$ orders of magnitude less than that of thermoelectric material. Therefore the boundary condition of electric current density at the rim of the hole is assumed to be impermeable. However, the heat conductivity of thermoelectric material is only 10 100 times more than that of air, and the influence of thermal conductivity of air inside the elliptic hole should be assessed. 
Since the electric impermeable elliptic hole boundary condition is adopted, the potential function of electric field $f_{1}(z)$ can be used the result in [21],

$$
f_{1}(z)=\frac{i j_{e 0} R}{\gamma}\left(\frac{1}{\zeta}-\zeta\right)
$$

Integrating Eq. (15) gives

$$
f_{2}(z)=\frac{i j_{e 0} R^{2}}{\gamma}\left[(m+1) \ln \zeta+\frac{1}{2 \zeta^{2}}-\frac{m \zeta^{2}}{2}\right]
$$

\subsection{The temperature field inside the hole}

The temperature field inside the elliptic hole has the form of

$$
T_{h}=\operatorname{Re}\left[g_{h}(z)\right]
$$

where $g_{h}(z)$ is an analytic function. Then the heat flux inside the hole can be obtained as

$$
q_{h x}-i q_{h y}=-k_{h} g_{h}^{\prime}(z)
$$

where $k_{h}$ is the heat conductivity of air. Using Gauss' law, we have

$$
\operatorname{Im}\left[k_{h} g_{h}(z)\right]=-\int q_{h n}(s) d s
$$

where $q_{h n}(s)$ represents the normal heat flux for the point $s$ along the boundary. The function $g_{h}(z)$ can be expressed in the form of the Faber series as [42, 43],

$$
g_{h}(z)=\sum_{n=1}^{\infty} a_{n}^{h}\left(m^{n} \zeta^{n}+\zeta^{-n}\right)
$$

where $a_{n}^{h}$ are complex coefficients to be determined.

\subsection{The temperature field in the thermoelectric material}

Inside the thermoelectric material, the complex temperature function $g(z)$ takes the form 


$$
g(z)=c_{2}^{\infty} z^{2}+c_{3}^{\infty} z+g_{0}(z)
$$

where $c_{2}^{\infty}$ and $c_{3}^{\infty}$ are two complex constants related to the loading condition at infinity, and $g_{0}^{\prime}(\infty)=0$.

Substituting Eqs. (15) and (21) into Eq. (6) 2, and then taking the limit $z \rightarrow \infty$ leads to:

$$
c_{2}^{\infty}=\frac{j_{e 0}^{2}}{4 k \gamma}, \quad c_{3}^{\infty}=i \frac{j_{u 0}}{k}
$$

To find $g_{0}(z)$, one has to use the continuous conditions of the normal component of heat flux and temperature on the hole. Noting that on the hole, $j_{e n}(s)=0$ and $\zeta=\sigma=e^{i \theta}$, these conditions require from Eqs. $(5)_{2},(7)_{2}$, (17) and (19) that

$$
\begin{gathered}
\operatorname{Im}\left[\frac{\gamma}{4} f_{1}^{2}(\sigma)+k g(\sigma)\right]=\operatorname{Im}\left[k_{h} g_{h}(\sigma)\right] \\
-\frac{\gamma}{4 k} f_{1}(\sigma) \overline{f_{1}(\sigma)}+\operatorname{Re}[g(\sigma)]=\operatorname{Re}\left[g_{h}(\sigma)\right]
\end{gathered}
$$

Substituting Eqs. (15), (20) and (21) into Eqs. (23) and (24) yields, and then multiplying both sides of them by $d \sigma /[2 \pi i(\sigma-\zeta)]$, one can obtain after calculating the Cauchy integration that [41]

$$
\begin{gathered}
g_{0}(\zeta)-\overline{g_{0}(0)}=-\frac{j_{e 0}^{2} R^{2}}{4 k \gamma}\left(m^{2}-1\right) \zeta^{2}-\frac{i j_{u 0} R}{k}(m+1) \zeta+\frac{k_{h}}{k} \sum_{n=1}^{\infty}\left[a_{n}^{h} m^{n} \zeta^{n}-\overline{a_{n}^{h}} \zeta^{n}\right] \\
g_{0}(\zeta)+\overline{g_{0}(0)}=-\frac{j_{e 0}^{2} R^{2}}{4 k \gamma}\left[\left(m^{2}+3\right) \zeta^{2}+4(m-1)\right]-\frac{i j_{u 0} R}{k}(m-1) \zeta+\sum_{n=1}^{\infty}\left[a_{n}^{h} m^{n} \zeta^{n}+\overline{a_{n}^{h}} \zeta^{n}\right]
\end{gathered}
$$

To find $a_{n}^{h}$ and $\overline{g_{0}(0)}$, substituting Eq. (25) into (26), and then equating the coefficients of the same power $\xi^{n}$ in both sides of (26), one has 


$$
\begin{gathered}
\overline{g_{0}(0)}=\frac{j_{e 0}^{2} R^{2}}{2 k \gamma}(1-m) \\
a_{2}^{h}=\frac{j_{e 0}^{2} R^{2}\left(m^{2}+1\right)}{2 \gamma\left[k_{h}\left(m^{2}-1\right)-k\left(m^{2}+1\right)\right]} \\
a_{1}^{h}=\frac{2 i j_{u 0} R}{k_{h}(m+1)-k(m-1)} \\
a_{n}^{h}=0,(n \geq 3)
\end{gathered}
$$

As a consequence, the solution of $g_{0}(\zeta)$ can be written as

$$
g_{0}(\zeta)=\left[k_{h} a_{2}^{h}-\frac{j_{e 0}^{2} R^{2}}{4 \gamma}\right] \frac{\left(m^{2}-1\right)}{k} \zeta^{2}+\left[k_{h} a_{1}^{h}-i j_{u 0} R\right] \frac{(m+1)}{k} \zeta-\frac{j_{e 0}^{2} R^{2}}{2 \gamma}(m-1)
$$

Finally, we have

$$
g(z)=\frac{j_{00}^{2} R^{2}}{4 k \gamma}\left(\frac{1}{\zeta}+\zeta\right)^{2}+\frac{i j_{u 0} R}{k}\left(\frac{1}{\zeta}-\zeta\right)+\frac{k_{h}}{k}\left[\left(m^{2}-1\right) a_{2}^{h} \zeta^{2}+(m+1) a_{1}^{h} \zeta\right]
$$

\subsection{Thermally and electrically induced elastic field}

Considering the condition of single-valued elastic displacement and stress, the complex potentials $\varphi(z)$ and $\phi(z)$ in the thermoelectric material have the form of

$$
\begin{aligned}
& \varphi(z)=A(\zeta) \ln \zeta+\varphi_{0}(z) \\
& \phi(z)=B(\zeta) \ln \zeta+\phi_{0}(z)
\end{aligned}
$$

where $A(\zeta)$ and $B(\zeta)$ are two unknown functions. Substituting Eq. (33) into Eqs. (9) and (12), the elastic displacement and resultant force must return the starting value when moving round the elliptic hole. These single-value conditions require

$$
\begin{gathered}
A=(m+1) A_{0} R^{2}\left[1-\frac{2 k_{h}}{k_{h}(m+1)-k(m-1)}\right] \\
B(\zeta)=\bar{A}+2(m+1) R^{3} P_{0}\left(\frac{1}{\zeta}-\zeta\right)
\end{gathered}
$$


with $A_{0}=-\frac{2 \mu \alpha^{*} i j_{u 0}}{k(\kappa+1)}$ and $P_{0}=\frac{\mu \alpha^{*} j_{e 0}^{2}}{2 k \gamma(\kappa+1)}$

The external forces on the rim of elliptic hole may be let zero for the thermoelectric material problem since the principle of superposition is applicable. Noting that on the elliptic hole, $\zeta=\sigma=e^{i \theta}$, the stress boundary condition (12) can be expressed as

$$
\varphi(\sigma)+\frac{\omega(\sigma)}{\overline{\omega^{\prime}(\sigma)}} \overline{\varphi^{\prime}(\sigma)}+\overline{\phi(\sigma)}=-\frac{\mu \alpha^{*} \gamma}{k(\kappa+1)} f_{2}(\sigma) \overline{f_{1}(\sigma)}
$$

Substituting Eqs. (13), (15), (16) and (33)-(35) into Eq. (36), and multiplying both sides of Eq. (36) by $d \sigma /[2 \pi i(\sigma-\zeta)]$ and carrying out the Cauchy integration yields [41],

$$
\varphi_{0}(\zeta)=m \bar{A}+m R^{3} P_{0} \zeta\left(\zeta^{2}-1\right)-\overline{\phi_{0}(0)}
$$

Taking the conjugate form of Eq. (36), multiplying by $d \sigma /[2 \pi i(\sigma-\zeta)]$ and carrying out the Cauchy integration, $\phi_{0}(z)$ is obtained as

$$
\phi_{0}(\zeta)=-\frac{m+\zeta^{2}}{m \zeta^{2}-1} A+R^{3} P_{0} \zeta\left(\zeta^{2}-1\right)-\frac{\zeta\left(m+\zeta^{2}\right)}{m \zeta^{2}-1} \varphi_{0}^{\prime}(\zeta)
$$

From Eq. (38), we have $\overline{\phi_{0}(0)}=m \bar{A}$. Finally, the complex stress potentials $\varphi(z)$ and $\phi(z)$ have the form of

$$
\begin{gathered}
\varphi(z)=A \ln \zeta+m R^{3} P_{0} \zeta\left(\zeta^{2}-1\right) \\
\phi(z)=\left[\bar{A}+2(m+1) R^{3} P_{0}\left(\frac{1}{\zeta}-\zeta\right)\right] \ln \zeta-\frac{m+\xi^{2}}{m \zeta^{2}-1} A \\
+\frac{R^{3} P_{0} \zeta}{m \zeta^{2}-1}\left[m^{2}+1-\left(3 m^{2}+1\right) \xi^{2}-2 m \xi^{4}\right]
\end{gathered}
$$




\section{The solution to an elliptic rigid inclusion}

Consider an infinite thermoelectric material containing an elliptic rigid inclusion with the major axis $2 a$ and the minor axis $2 b$ in the $z$-plane as shown in Fig. 1 . The uniform thermoelectric loadings $j_{e 0}$ and $j_{u 0}$ are simultaneously applied at infinity. The inclusion is assumed to be electrical insulted, i.e., $\gamma_{i}=0$, but its heat conductivity $k_{i}$ is not zero. In addition, the bonds between the thermoelectric material and the inclusion are assumed to be perfect along the common boundary. The mapping function is expressed by Eq. (13), and the electric potential and temperature for such a thermoelectric boundary condition is given by Eqs. (15) and (32). The corresponding displacement boundary condition is,

$$
\kappa \varphi(\sigma)-\frac{\omega(\sigma)}{\overline{\omega^{\prime}(\sigma)}} \overline{\varphi^{\prime}(\sigma)}-\overline{\phi(\sigma)}+2 \mu \alpha^{*} \int g(\sigma) \omega^{\prime}(\sigma) d \sigma-\frac{\mu \alpha^{*} \gamma}{k(\kappa+1)} f_{2}(\sigma) \overline{f_{1}(\sigma)}=0
$$

The stress potential functions can be determined as the similar procedures of elliptic hole problem, and the expressions of $\varphi_{0}(\zeta)$ and $\phi_{0}(\zeta)$ are obtained as:

$$
\begin{aligned}
\kappa \varphi_{0}(\zeta)= & -(\kappa+1) P_{0} R^{3} \zeta\left(\frac{m}{3} \zeta^{2}-2 m-1\right)-\frac{\kappa+1}{2} A_{0} m R^{2} \zeta^{2}-P_{0} R^{3} m \zeta\left(\zeta^{2}-1\right) \\
& -2 \mu \alpha^{*} \frac{k_{i} R a_{2}^{i}}{k}\left(m^{2}-1\right) \zeta\left(\frac{m}{3} \zeta^{2}-1\right)-\mu \alpha^{*} \frac{k_{i} R a_{1}^{i}}{k} m(m+1) \zeta^{2} \\
\phi_{0}(\zeta)= & \frac{m+\zeta^{2}}{m \zeta^{2}-1} A-(\kappa+1) P_{0} R^{3} \zeta\left(\frac{m}{3} \zeta^{2}-m+2\right)-\frac{\kappa+1}{2} A_{0} R^{2} \zeta^{2} \\
& -P_{0} R^{3} \zeta\left(\zeta^{2}-1\right)+\frac{\zeta\left(m+\zeta^{2}\right)}{m \zeta^{2}-1} \varphi_{0}^{\prime}(\zeta)
\end{aligned}
$$

where

$$
\begin{gathered}
a_{2}^{i}=\frac{j_{e 0}^{2} R^{2}\left(m^{2}+1\right)}{2 \gamma\left[k_{i}\left(m^{2}-1\right)-k\left(m^{2}+1\right)\right]} \\
a_{1}^{i}=\frac{2 i j_{u 0} R}{k_{i}(m+1)-k(m-1)}
\end{gathered}
$$




\section{Numerical results and discussion}

In this section, the influences of applied electric current density and energy flux, heat conductivity of medium inside the elliptic hole/inclusion on the temperature field and stress components are investigated, respectively. The thermoelectric material is considered to be $\mathrm{Bi}_{2} \mathrm{Te}_{3}$, and its electric conductivity $\gamma=1.1 \times 10^{5} \mathrm{Sm}^{-1}$, heat conductivity $k=1.6 \mathrm{Wm}^{-1} \mathrm{~K}^{-1}$, Seebeck coefficient $\varepsilon=2 \times 10^{-4} V K^{-1}$ [44], and Poisson's ratio $v=0.4$ [45]. The effect of $j_{e 0}$ on hoop electric field $j_{e \theta}$ and $\sigma_{\theta \theta}$ around an electrical impermeable elliptic hole was studied in our previous work [21], and does not discuss it again in this paper. The energy flux and stress components $\sigma_{r r}$ and $\sigma_{\theta \theta}$ in the curvilinear coordinates $(r, \theta)$ expressed by the mapping function are

$$
\begin{gathered}
j_{u r}-i j_{u \theta}=\frac{\zeta \omega^{\prime}(\zeta)}{\left|\zeta \omega^{\prime}(\zeta)\right|}\left[j_{u r}-i j_{u \theta}\right]=-\frac{\zeta}{\left|\zeta \omega^{\prime}(\zeta)\right|}\left[\frac{\gamma}{2} f_{1}(\zeta) f_{1}^{\prime}(\zeta)+k g^{\prime}(\zeta)\right] \\
\sigma_{r r}+\sigma_{\theta \theta}=\sigma_{x x}+\sigma_{y y}=4 \operatorname{Re}\left[\frac{\varphi^{\prime}(\zeta)}{\omega^{\prime}(\zeta)}\right]+\frac{2 \mu \alpha^{*} \gamma}{k(\kappa+1)} f_{1}(\zeta) \overline{f_{1}(\zeta)}
\end{gathered}
$$

\subsection{Elliptic hole case}

First, a thermoelectric material with an elliptic cavity filled with air is considered, and the heat conductivity of air $k_{h}=0.024 W^{-1} K^{-1}$. From Eqs. (15), (32), (39), (46) and (47), we have

$$
\begin{gathered}
j_{u r}-i j_{u \theta}=\frac{\zeta}{\mid R \zeta\left(m-\zeta^{-2}\right)}\left\{i j_{u 0} R\left(1+\zeta^{-2}\right)-k_{h}\left[2\left(m^{2}-1\right) a_{2}^{h} \zeta+(m+1) a_{1}^{h}\right]\right\} \\
\sigma_{r r}+\sigma_{\theta \theta}=4 \operatorname{Re}\left[\frac{\zeta A+m R^{3} P_{0} \zeta^{2}\left(3 \zeta^{2}-1\right)}{R\left(m \zeta^{2}-1\right)}\right]+4 R^{2} P_{0}\left(\frac{1}{\zeta}-\zeta\right)\left(\frac{1}{\bar{\zeta}}-\bar{\zeta}\right)
\end{gathered}
$$


Noting that on the hole $\operatorname{rim}, \quad \zeta=\sigma=e^{i \theta}=\cos \theta+i \sin \theta, \quad j_{u \theta}$ and $\sigma_{\theta \theta}$ can be obtained as

$$
\begin{gathered}
j_{u \theta}=\frac{\left(m^{4}-1\right) k_{h} R j_{e 0}^{2} \sin \theta}{\gamma\left[\left(m^{2}-1\right) k_{h}-\left(m^{2}+1\right) k\right] \sqrt{m^{2}+1-2 m \cos (2 \theta)}} \\
+\frac{2(m-1) k j_{u 0} \cos \theta}{\left[(m+1) k_{h}-(m-1) k\right] \sqrt{m^{2}+1-2 m \cos (2 \theta)}} \\
\sigma_{\theta \theta}=\frac{4 m R^{2} P_{0}\left\{[m-\cos (2 \theta)][3 \cos (2 \theta)-1]+3 \sin ^{2}(2 \theta)\right\}}{m^{2}+1-2 m \cos (2 \theta)}+16 R^{2} P_{0} \sin ^{2} \theta \\
-\frac{4 i(m+1)^{2} R A_{0} \sin \theta}{m^{2}+1-2 m \cos (2 \theta)}\left[1-\frac{2 k_{h}}{k_{h}(m+1)-k(m-1)}\right]
\end{gathered}
$$

From the definition of energy flux and potential function $F$, the hoop heat flux $q_{\theta}$ on the hole rim has the form of

$$
\begin{aligned}
q_{\theta}= & j_{u \theta}+\frac{2 j_{e 0}^{2} R \sin (2 \theta)}{\gamma \sqrt{m^{2}+1-2 m \cos (2 \theta)}} \\
& -\frac{\varepsilon j_{e 0}^{3} R^{2}\left(m^{2}+1\right)^{2} \cos \theta \cos (2 \theta)}{\left[\left(m^{2}-1\right) k_{h}-\left(m^{2}+1\right) k\right] \sqrt{m^{2}+1-2 m \cos (2 \theta)}}
\end{aligned}
$$

From Eq. (52), we can see that $q_{\theta}=j_{u \theta}$ if $j_{e 0}=0$, the $j_{e 0}^{2}$ and $j_{e 0}^{3}$ terms are induced by the Joule heat and Seebeck effect, respectively. It is clear that constants $P_{0}$ and $A_{0}$ stand for the applied electric current density and energy flux from Eqs. (34) and (35), respectively. Stress field has a linear relationship with the remote applied energy flux, but has a non-linear relationship with the remote applied electric current density. The variations of normalized $j_{u \theta} / j_{u 0}$ and $\sigma_{\theta \theta} /\left(i R A_{0}\right)$ along the rim of the elliptic hole with $\theta$ for different $a / b$ are plotted in Figs. 2-3. It is found that the concentration factors of hoop energy flux and stress increase as $a / b$ increases, no matter if the heat conductivity of air inside the hole is or not assumed to be zero. The 
numerical results show that the temperature field within the elliptic hole can be neglected for the case of $a / b \leq 2$, that is, the heat impermeable boundary condition can be used to simplify the analysis. However, as $a / b$ increases, the effect of $k_{h}$ on hoop energy flux and stress should be considered by comparing the results obtained based on the heat exact boundary condition and heat insulted model, respectively. Figs. 4 and 5 show that the effect of applied electric current density $j_{e 0}$ on the normalized hoop energy flux $j_{u \theta}$ and hoop heat flux $q_{\theta}$ for the different value of major to minor axis ratio of the elliptic hole. Generally, the distribution of temperature field is affected by the heat conductivity of air inside the elliptic hole $k_{h}$ due to thermoelectric coupling effect even if the electric current density $j_{e 0}$ is solely applied.

\subsection{Elliptic rigid inclusion case}

Further, to study the effects of thermoelectric loadings on mechanical behavior of elliptic rigid inclusion problem, the hoop stress along the elliptic rigid inclusion boundary can be calculated from Eqs. $(33)_{1},(42)$ and (47), and it is very complicate. In the following analysis, the hoop stress is given for the case of $j_{e 0}=0$ to illustrate the influence of heat conductivity of inclusion and $a / b$ on $\sigma_{\theta \theta}$,

$$
\begin{array}{r}
\sigma_{\theta \theta}=-\frac{4 i(m+1) R A_{0}}{m^{2}+1-2 m \cos (2 \theta)}\left\{\frac{m+1}{\kappa+1}\left[1-\frac{2 k_{i}}{k_{i}(m+1)-k(m-1)}\right] \sin \theta\right. \\
\left.+\frac{1}{\kappa}\left[\frac{2 m k_{i}}{k_{i}(m+1)-k(m-1)}-\frac{m}{m+1}\right][\sin (3 \theta)-m \sin \theta]\right\}
\end{array}
$$

The expression of hoop energy flux at the elliptic inclusion faces agrees with Eq. (50). Figs. 6-8 show that $k_{i}$ has great influence on the hoop energy flux and stress at the inclusion faces when the remote energy flux is applied. It is found that the results 
based on the exact boundary condition show the hoop component of heat flux decreases with the increasing value of $k_{i} / k$ for $a / b=10$ as shown in Fig. 6. However, unlike the elliptic cavity problem, Figs. 7 and 8 show that the concentration factors of hoop stress increase as the heat conduction coefficient of inclusion increasing for a given thermoelectric loading and $a / b$. The reason is that the displacement on the common boundary between the thermoelectric medium and inclusion caused by the temperature change is completely prevented since the elliptic inclusion is assumed to be absolutely rigid and the common interface is perfectly bonded. It is shown from Figs. 9 and 10 that energy flux and heat flux inside the inclusion is not zero when the heat exact boundary condition is used for a given electric current density due to the Joule heat and Seebeck effect. When $j_{e 0}$ exerted at infinity, the influence of $k_{i} / k$ is very small on $\sigma_{\theta \theta}$ on the rim of inclusion (Fig. 11). Especially for $a / b=1$ (a circular inclusion), the heat conductivity of the rigid inclusion can be neglected.

\section{Conclusion}

The exact solution for an elliptic hole or rigid inclusion embedded in an infinite thermoelectric material subjected to far-field uniform energy flux and electric current density loading is presented in this paper. The derivation is based on the complex conformal mapping technique, electric insulated and heat exact boundary conditions on the rim of the hole or inclusion. The complex potentials in the thermoelectric medium and temperature field inside the hole or inclusion are obtained in closed-form, respectively. Particular attention is given to the effect of heat conduction coefficient inside the elliptic hole or the rigid inclusion on the hoop energy flux and stress distribution. For the elliptic hole case, it is found that the influence of heat 
conductivity of air is very limited. It can be neglected especially when $a / b \leq 2$. However, for the elliptic rigid inclusion case, the heat conductivity of inclusion has a great influence on the hoop energy flux and stress in thermoelectric material when the energy flux is exerted at infinity. In addition, the stress concentration factor increases as the heat conduction coefficient of rigid inclusion increases. This paper presents a theoretical basis on studying the effective behavior of thermoelectric composite materials with cracks for their design and optimization.

Finally, the effect of electric permeability of an elliptic rigid inclusion on the fracture behavior of thermoelectric materials should be considered if the metallic inclusion is introduced. Here, some brief discussions are given. The electric current density, electric potential and temperature fields across the interface between the matrix and inclusion are continuous, therefore, the electric boundary condition can be expressed as,

$$
\begin{aligned}
& \gamma\left[f_{1}(z)-\overline{f_{1}(z)}\right]=\gamma_{i}\left[f_{i}(z)-\overline{f_{i}(z)}\right] \\
& \operatorname{Re}\left[f_{1}(z)\right]=\operatorname{Re}\left[f_{i}(z)\right]-\frac{\varepsilon \gamma_{i}}{8 k_{i}}\left[f_{i}(z)+\overline{f_{i}(z)}\right]^{2}+\varepsilon \operatorname{Re}\left[g_{i}(z)\right]
\end{aligned}
$$

where $\gamma_{i}$ is the electric permeability of the inclusion, and $f_{i}(z)$ is the electric potential function to be determined by the boundary conditions. The subsequent task is to obtain the temperature and stress fields, which is similar to the analysis in Section 3.

Acknowledgements : The research was supported by the National Science Foundation of China (NSFC) (Project Nos. 11402063, 11672084 and 11372086), the Natural Science Foundation of Zhejiang Province of China (LY17A020001), the Natural Science Foundation of Guangdong Province of China (2016A030311006), Research 
Innovation Fund of Shenzhen City of China (project Nos. JCYJ20150805142729431, JCYJ2016042718464530), Research Fund for the Doctoral Program of Higher Education of China (No. 20123305110006), Zhejiang Provincial Top Key Discipline of Mechanics Open Foundation (xklx1604) and the K. C. Wong Magna Fund in Ningbo University.

\section{References}

[1] T.M. Tritt, M.A. Subramanian, Thermoelectric materials, phenomena, and applications: a bird's eye view, MRS Bullet. 31 (2006) 188-194.

[2] D. Narducci, Do we really need high thermoelectric figures of merit? A critical appraisal to the power conversion efficiency of thermoelectric materials, Appl. Phys. Lett. 99 (2011) 102104.

[3] D. Kraemer, B. Poudel, H.P. Feng, J.C. Caylor, B. Yu, X. Yan, Y. Ma, X.W. Wang, D.Z. Wang, A. Muto, K. Mcenaney, M. Chiesa, Z.F. Ren, G. Chen, High-performance flat-panel solar thermoelectric generators with high thermal concentration, Nat. Mater. 10 (2011) 532-538.

[4] L.E. Bell, Cooling, heating, generating power, and recovering waste heat with thermoelectric systems, Science 321 (2008) 1457-1461.

[5] F. Ren, H. Wang, P.A. Menchhofer, J.O. Kiggans, Thermoelectric and mechanical properties of multi-walled carbon nanotube doped $\mathrm{Bi}_{0.4} \mathrm{Sb}_{1.6} \mathrm{Te}_{3}$ thermoelectric material, Appl. Phys. Lett. 103 (2013) 221907.

[6] R.D. Schmidt, X.F. Fan, E.D. Case, P.B. Sarac, Mechanical properties of $\mathrm{Mg}_{2} \mathrm{Si}$ thermoelectric materials with the addition of $0-4$ vol\% silicon carbide nanoparticles (SiCNP), J. Mater. Sci. 50 (2015) 4034-4046.

[7] Z.G. Zhou, L.Z. Wu, B. Wang, The behavior of a crack in functionally graded 
piezoelectric/piezomagnetic materials under anti-plane shear loading. Arch. Appl. Mech. 74 (2005) 526-535.

[8] L.C. Guo, L.Z. Wu, T. Zeng, Mode I crack problem for a functionally graded orthotropic strip, Eur. J. Mech. A-Solid 23 (2004) 219-234.

[9] W.G. Li, D.J. Li, C.Z. Zhang, D.N. Fang, Modelling the effect of temperature and damage on the fracture strength of ultra-High temperature ceramics, Int. J. Fract. 176 (2012) 181-188.

[10] H.P. Wu, L. Li, G.Z. Chaia, F. Songc, T. Kitamura, Three-dimensional thermal weight function method for the interface crack problems in bimaterial structures under a transient thermal loading, J. Thermal Stresses 39 (2016) 371-385.

[11] Z.G. Zhou, P.W. Zhang, L.Z. Wu, The closed form solution of a Mode-I crack in the piezoelectric/piezomagnetic materials. Int. J. Solids Struct. 44 (2007) 419-435.

[12] L. Ma, L.Z. Wu, Z.G. Zhou, L.C. Guo, Fracture analysis of a functionally graded piezoelectric strip, Compos. Struct. 69 (2005) 294-300.

[13] M. Shi, H.P. Wu, L. Li, G.Z. Chai, Calculation of stress intensity factors for functionally graded materials by using the weight functions derived by the virtual crack extension technique. Int. J. Mech. Mater. Des. 10 (2014) 65-77.

[14] G.C. Yu, L.Z. Wu, L.J. Feng, W. Yang, Thermal and mechanical properties of carbon fiber polymer-matrix composites with a 3D thermal conductive pathway. Compos. Struct. 149 (2016) 213-219.

[15] Y.Y. Feng, L.Z. Wu, Analysis of interfacial thermal stresses of chip-substrate structure. Int. J. Solids Struct. 38 (2001) 1551-1562.

[16] L.C. Guo, L.Z. Wu, T. Zeng, L. Ma, The dynamic fracture behavior of a functionally graded coating-substrate system. Compos. Struct. 64 (2004) 
433-441.

[17] R.D. Schmidt, E.D. Case, III.J. Giles, J.E. NI, T.P. Hogan, Room-Temperature mechanical properties and slow crack growth behavior of $\mathrm{Mg}_{2} \mathrm{Si}$ thermoelectric materials, J. Electron. Mater. 41 (2012) 1210-1216.

[18] A.B. Zhang, B.L. Wang, Crack tip field in thermoelectric media, Theor. Appl. Fract. Mech. 66 (2013)33-36.

[19] H.P. Song, C.F. Gao, J.Y. Li, Two-dimensional problem of a crack in thermoelectric materials, J. Thermal Stresses 38 (2015) 325-337.

[20] G.D. Li, Q. An, W.A. Goddard III, R. Hanus, P.C. Zhai, Q.J. Zhang, G.J. Snyder, Atomistic explanation of brittle failure of thermoelectric skutterudite $\mathrm{CoSb}_{3}$, Acta Mater. 103 (2016) 775-780.

[21] A.B. Zhang, B.L. Wang, Explicit solutions of an elliptic hole or a crack problem in thermoelectric materials, Eng. Fract. Mech. 151 (2016) 11-21.

[22] X.C. Zhong, K.Y. Lee, A thermal-medium crack model, Mech. Mater. 51 (2012) $110-117$.

[23] A.B. Zhang, B.L. Wang, Temperature and electric potential fields of an interface crack in a layered thermoelectric or metal/thermoelectric material, Int. J. Therm. Sci. 104 (2016) 396-403.

[24] J.H. Bahk, Z.X. Bian, M. Zebarjadi, J.M.O. Zide, H. Lu, D.Y. Xu, P. Feser, G.H. Zeng, A. Majumdar, A.C. Gossard, A. Shakouri, J.E. Bowers, Thermoelectric figure of merit of $\left(\operatorname{In}_{0.53} \mathrm{Ga}_{0.47} \mathrm{As}\right)_{0.8}\left(\mathrm{In}_{0.52} \mathrm{Al}_{0.48} \mathrm{As}\right)_{0.2} \mathrm{III}-\mathrm{V}$ semiconductor alloys, Phys. Rev. B 81 (2010) 235209.

[25] A.U. Khan, N. Vlachos, T. Kyratsi, High thermoelectric figure of merit of $\mathrm{Mg}_{2} \mathrm{Si}_{0.55} \mathrm{Ge}_{0.05}$ materils doped with $\mathrm{Bi}$ and $\mathrm{Sb}$, Csirpta Mater. 69 (2013) 606-609. 
[26] D. Kraemer, J. Sui, K. McEnaney, H. Zhao, Q. Jie, Z.F. Ren, G. Chen, High thermoelectric conversion efficiency of MgAgSb-based material with hot-pressed contacts, Energy Environ. Sci. 8 (2015) 1299-1308.

[27] G.J. Snyder, M. Christensen, E. Nishibori, T. Caillat, B.B. Iversen, Disordered zinc in $\mathrm{Zn}_{4} \mathrm{Sb}_{3}$ with phonon-glass and electron-crystal thermoelectric properties. Nat. Mater. 3 (2004) 458-463.

[28] M.S. Dresselhaus, G. Chen, M.Y. Tang, R.G. Yang, H. Lee, D.Z. Wang, Z.F. Ren, J.P. Fleurial, P. Gogna, New Directions for low-dimensional thermoelectric materials. Adv. Mater. 19 (2007) 1043-1053.

[29] W.S. Liu, X. Yan, G. Chen, Z.F. Ren, Recent advances in thermoelectric nanocomposites. Nano Energy 1 (2012) 42-56.

[30] T.M. Tritt, H. Boettner, L. Chen, Thermoelectric: direct solar thermal energy conversion. MRS Bullet. 33 (2008) 366-368.

[31] G.J. Snyder, E.S. Toberer, Complex thermoelectric materials. Nat. Mater. 7 (2008) 105-114.

[32] X.Y. Huang, Z. Xu, L.D. Chen, The thermoelectric performance of $\mathrm{ZrNiSn} / \mathrm{ZrO} 2$ composites. Solid State Commun. 130 (2004)181-185.

[33] Y. Yang, F.Y. Ma, C.H. Lei, Y.Y. Liu, J.Y. Li, Is thermoelectric conversion efficiency of a composite bounded by its constituents? Appl. Phys. Lett. 102 (2013) 053905.

[34] Y.Z. Wang, Effective material properties of thermoelectric composites with elliptical fibers, App. Phys. A 119 (2015) 1081-1085.

[35] S.V. Faleev, F. Léonard, Theory of enhancement of thermoelectric properties of materials with nanoinclusions. Phys. Rev. B 77 (2008) 214304.

[36] N. Wang, H. Chen, H. He, W. Norimatsu, M. Kusunoki, K. Koumoto, Enhavced 
thermoelectric performance of $\mathrm{Nb}$-doped $\mathrm{SrTiO}_{3}$ by nano-inclusion with low thermal conductivity. Sci. Rep. 3 (2013) 3449.

[37] M. Saleemi, A. Famengo, S. Fiameni, S. Boldrini, S. Battiston, M. Johnsson, M. Muhammed, M.S. Toprak, Thermoelectric performance of higher manganese silicide nanocomposites, J. Alloy. Compd. 619 (2015) 31-37.

[38] D.J. Bergman, L.G. Fel, Enhancement of thermoelectric power factor in composite thermoelectric, J. Appl. Phys. 85 (1999) 8205.

[39] D.J. Bergman, O. Levy, Thermoelectric properties of a composite medium, J. Appl. Phys. 70 (1991) 6821.

[40] K.Y. Lee, S.J. Park, Thermal stress intensity factors for partially insulated interface crack under uniform heat flow, Eng. Fract. Mech. 50 (1995) 475-82.

[41] N.I. Muskhelishvili, Some basic problems of the mathematical theory of elasticity, Noordhoff, Leiden, 1975.

[42] J.H. Cutis, Faber polynomials and the Faber series, Am. Math. Mon. 78 (1971) 577-596.

[43] A.S. Kosmodamianskii, V.I. Chemie, Stress state of a plate weakened by two elliptical holes with parallel axes, Int. Appl. Mech. 17 (1981) 570-581.

[44] X.D. Wang, Y.X. Huang, C.H. Cheng, C.T. Lin, C.H. Kang, A three-dimensional numerical modeling of thermoelectric device with consideration of coupling of temperature field and electric potential field, Energy 47 (2012) 488-497.

[45] J.L. Gao, Q.G. Du, X.D. Zhang, X.Q. Jiang, Thermal stress analysis and structure parameter selection for a $\mathrm{Bi}_{2} \mathrm{Te}_{3}$-based thermoelectric module, J. Electron. Mater. 40 (2011) 884-888. 


\section{List of figure captions}

Figure 1. Thermoelectric material with an elliptic hole or rigid inclusion for (a) $z$-plane and (b) $\zeta$-plane

Figure 2. Effect of heat conductivity of air and $a / b$ on the energy flux for the elliptic hole case

Figure 3. Effect of heat conductivity of air and $a / b$ on the hoop stress for the elliptic hole case

Figure 4. Effect of $a / b$ on the energy flux for the elliptic hole case

Figure 5. Effect of $a / b$ on the heat flux for the elliptic hole case

Figure 6. Effect of heat conductivity of inclusion on the energy flux for the elliptic rigid inclusion case $(a / b=10)$

Figure 7. Effect of heat conductivity of inclusion and $a / b$ on the hoop stress for the elliptic rigid inclusion case

Figure 8. Effect of heat conductivity of rigid inclusion on the hoop stress for the elliptic rigid inclusion case $(a / b=10)$

Figure 9. Effect of heat conductivity of rigid inclusion on the energy flux for the elliptic rigid inclusion case $(a / b=10)$

Figure 10. Effect of heat conductivity of rigid inclusion on the heat flux for the elliptic rigid inclusion case $(a / b=10)$

Figure 11. Effect of heat conductivity of rigid inclusion and $a / b$ on the hoop stress for the elliptic rigid inclusion case 


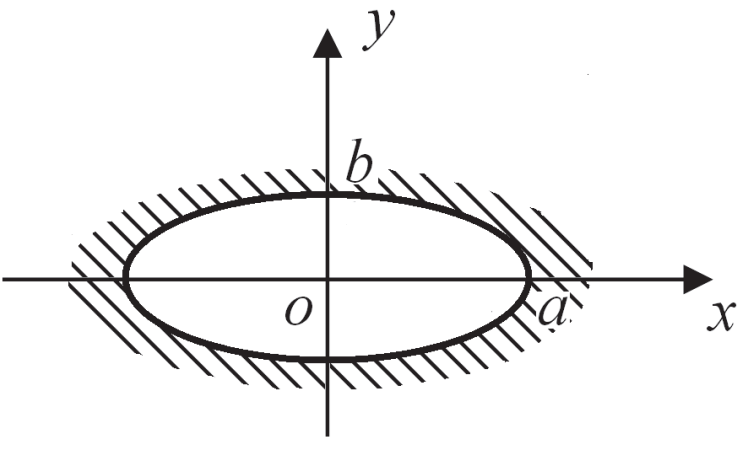

(a) z-plane

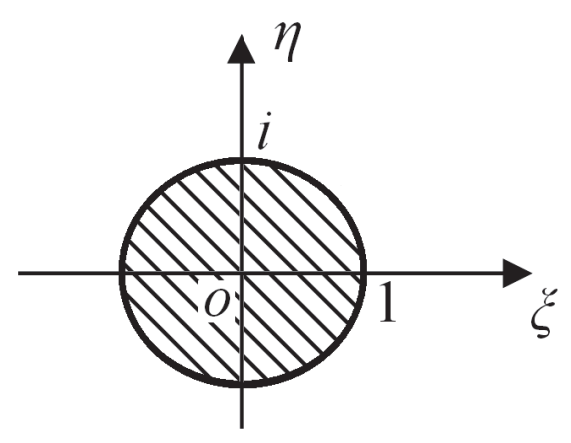

(b) ک-plane

Fig. 1 


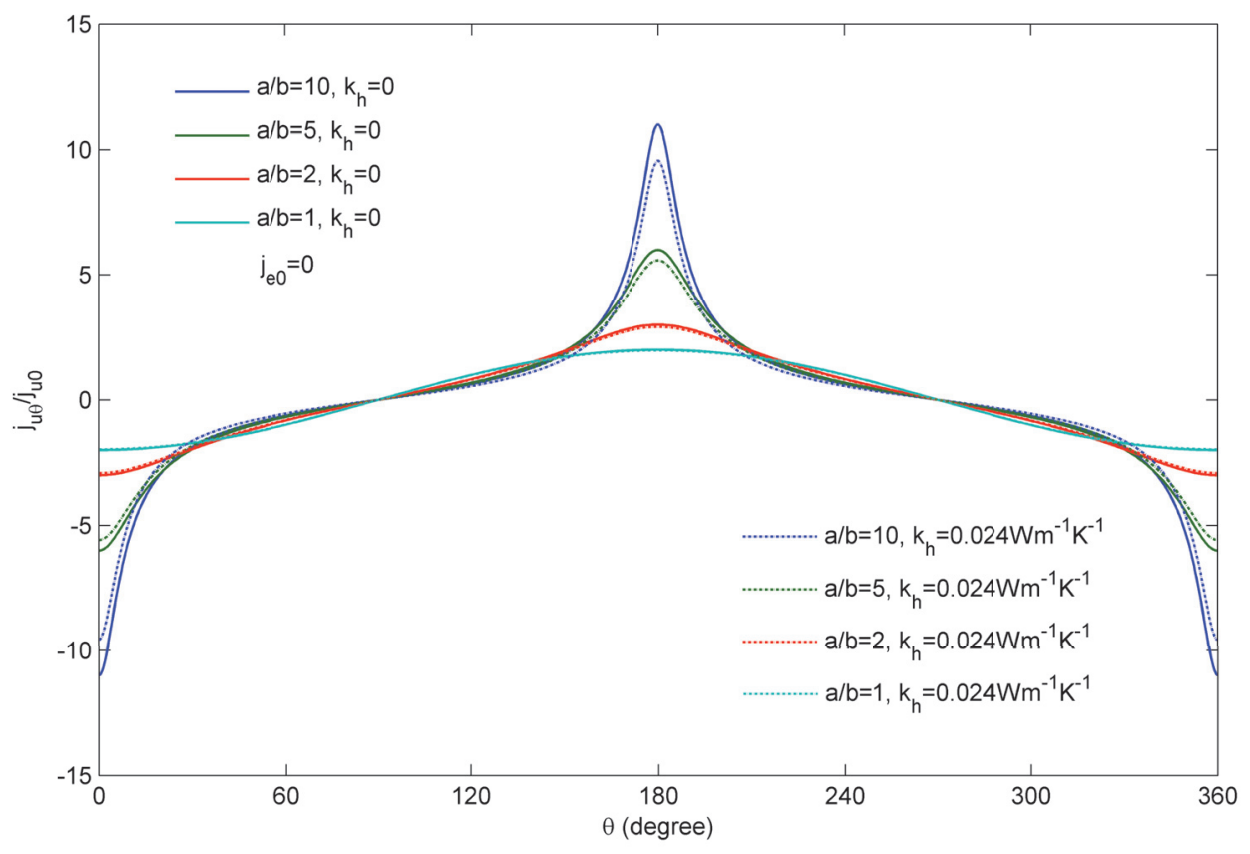

Fig. 2 


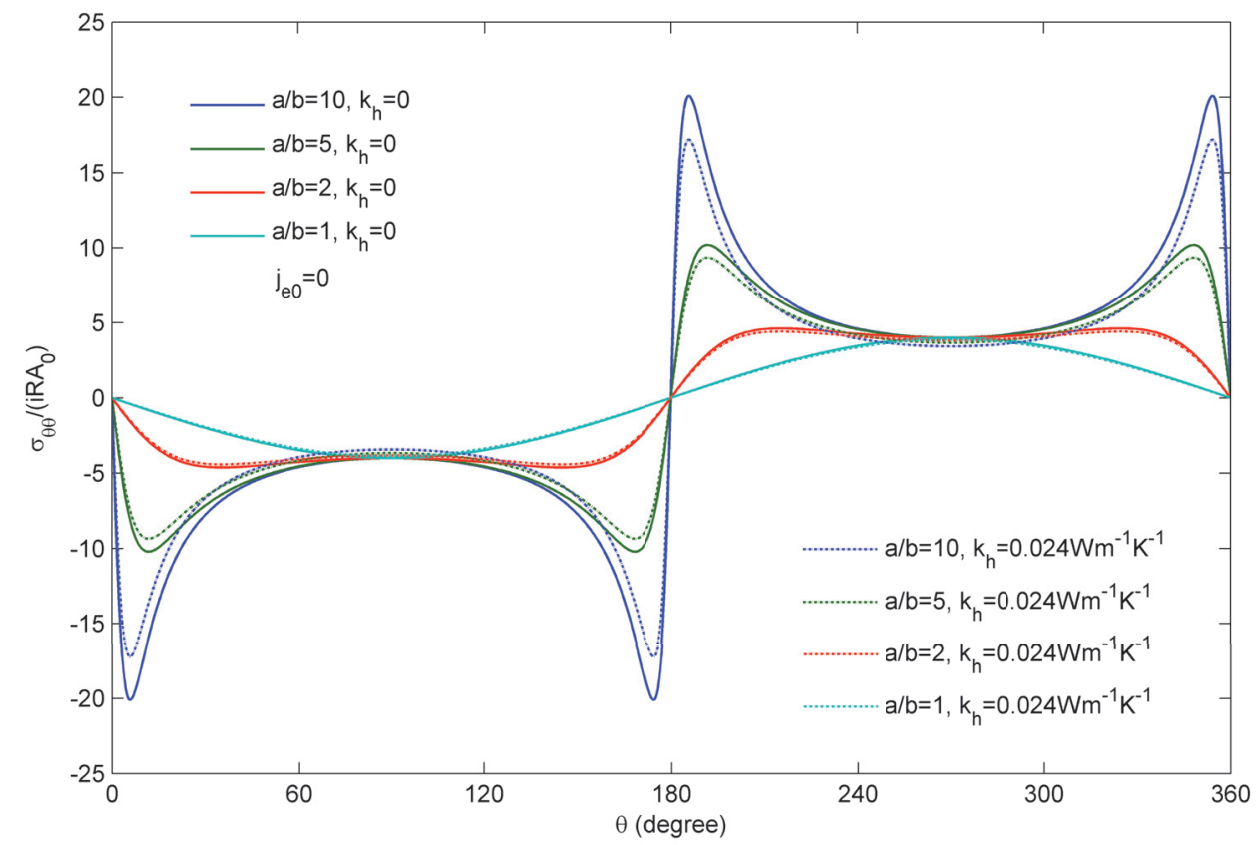

Fig. 3 


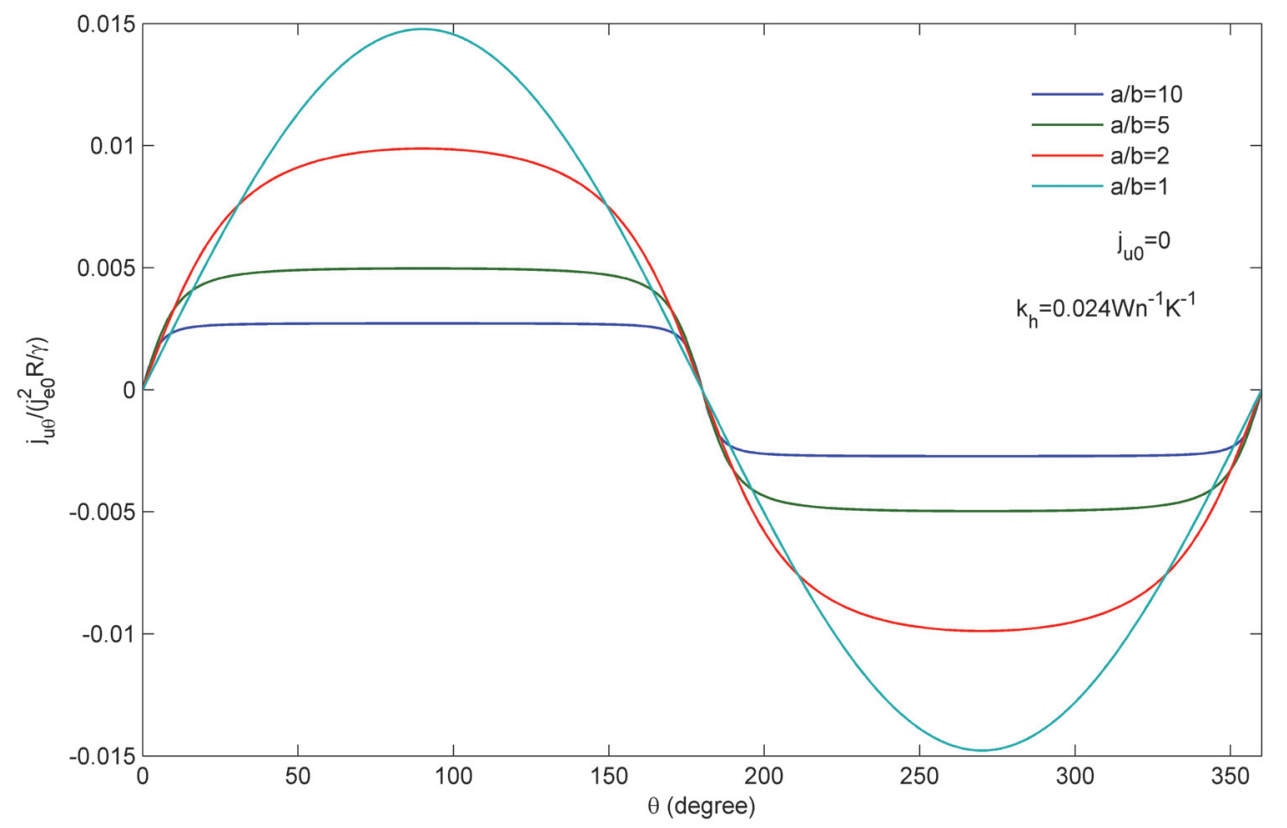

Fig. 4 


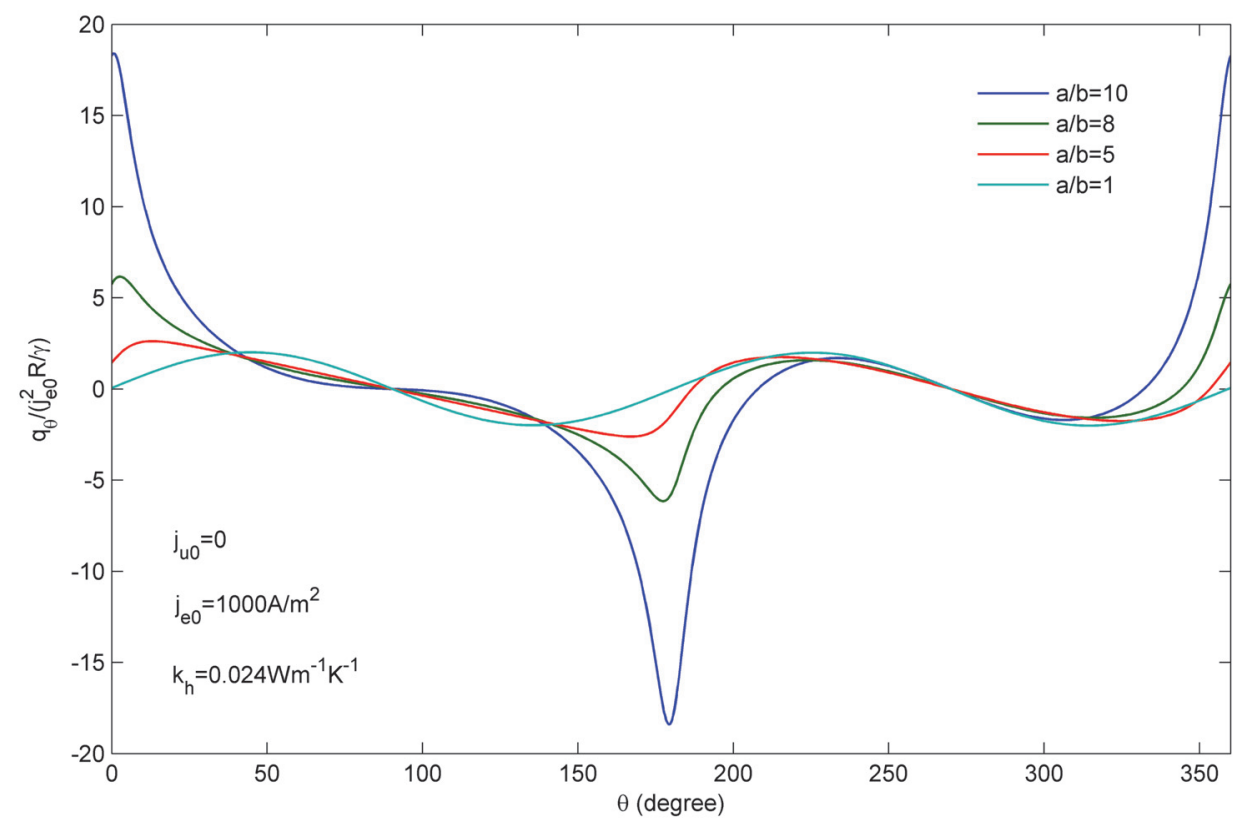

Fig. 5 


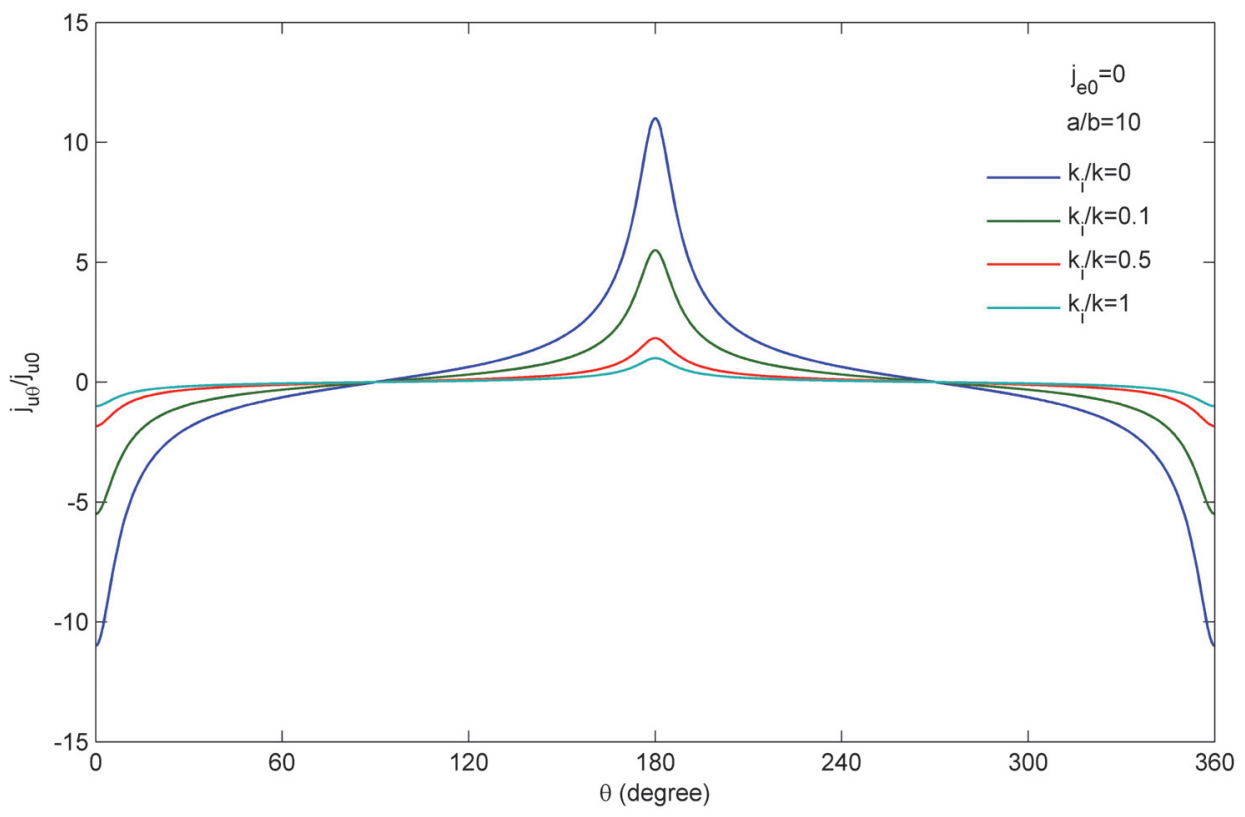

Fig. 6 


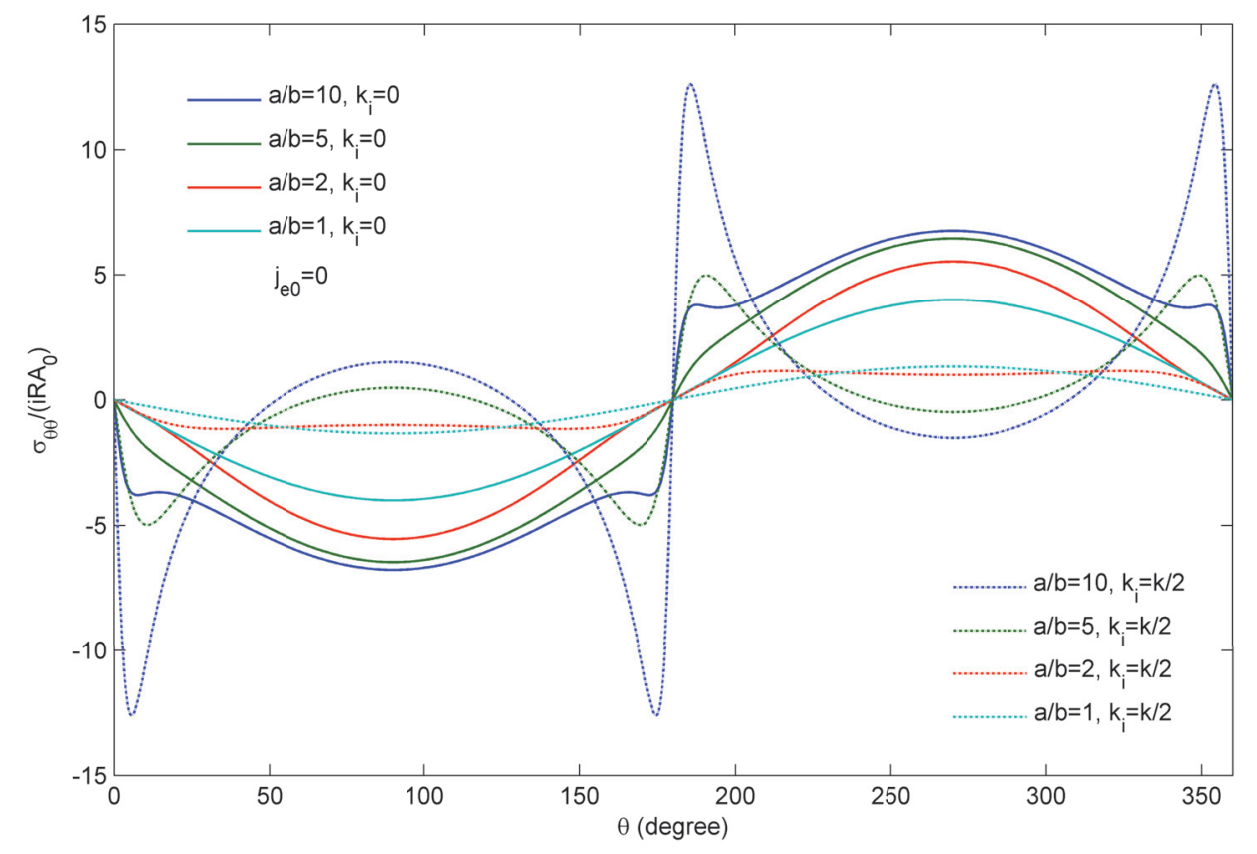

Fig. 7 


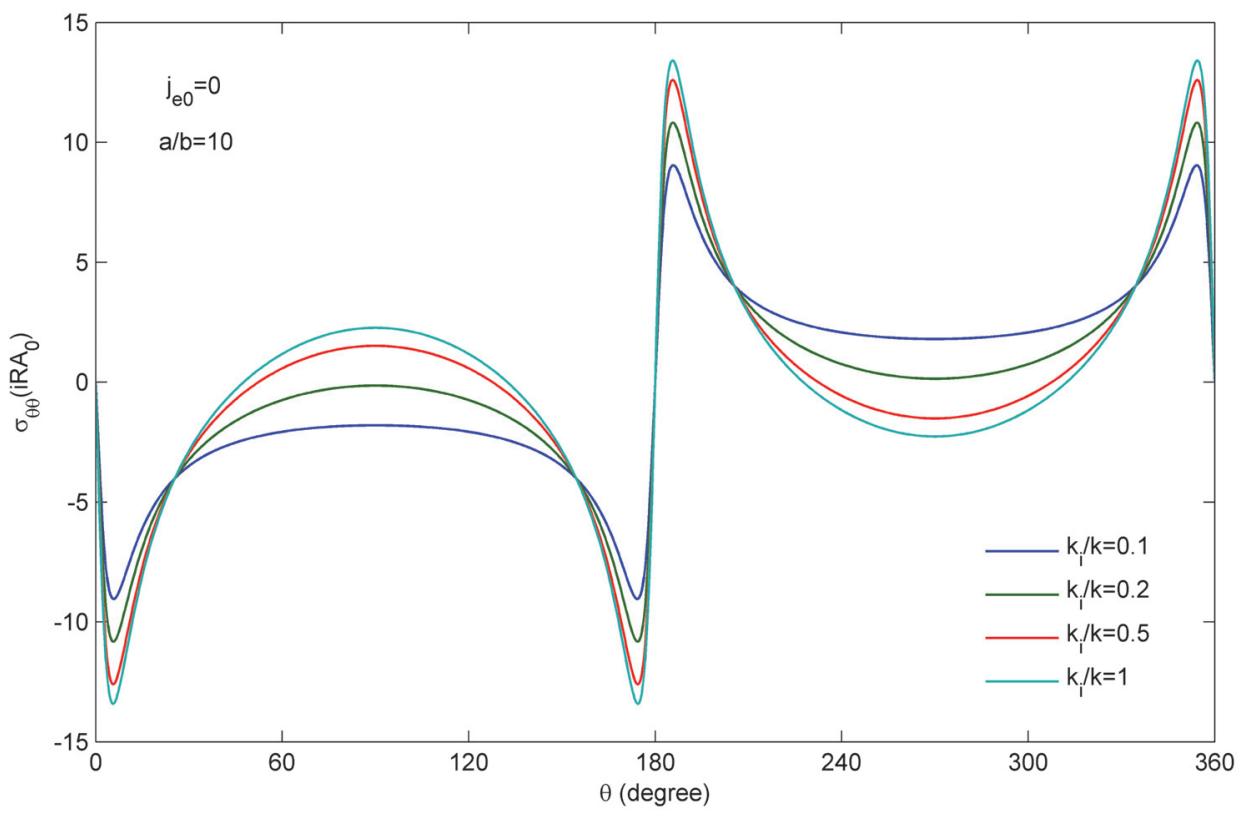

Fig. 8 


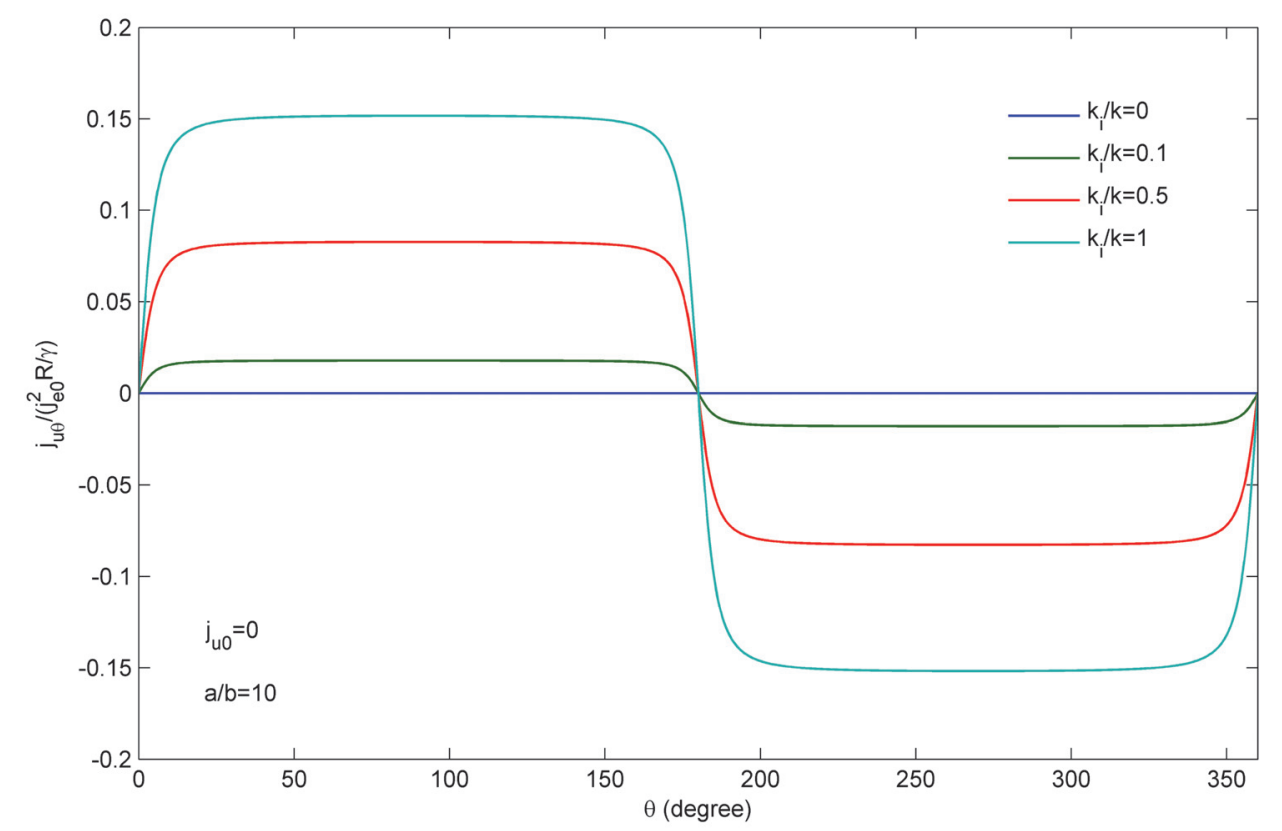

Fig. 9 


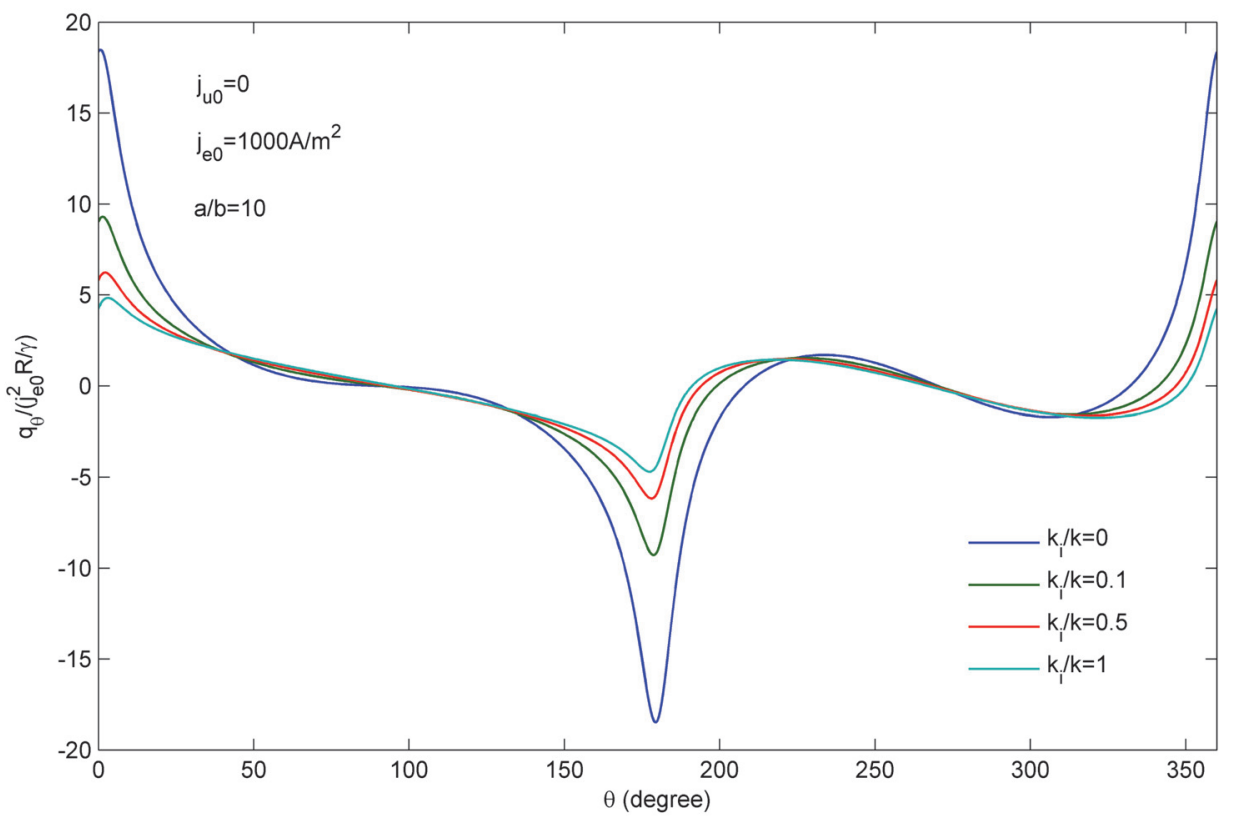

Fig. 10 


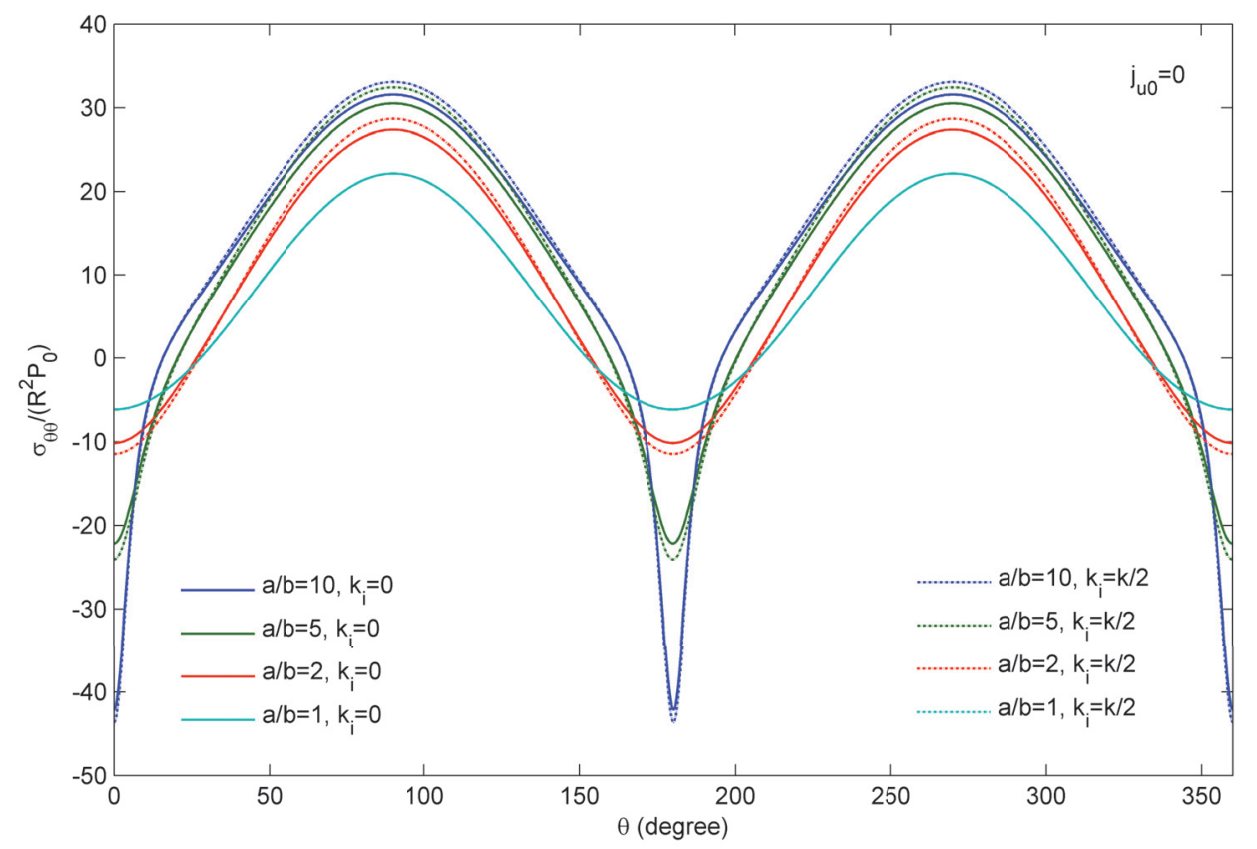

Fig. 11 